\title{
Development and validation of a predictive model for American Society of Anesthesiologists Physical Status
}

Seshadri C. Mudumbai ${ }^{1,2,3^{*}}$ (D), Suzann Pershing ${ }^{3,4}$, Thomas Bowe ${ }^{3}$, Robin N. Kamal ${ }^{3,5}$, Erika D. Sears ${ }^{6,7}$, Andrea K. Finlay ${ }^{3}$, Dan Eisenberg ${ }^{3,8}$, Mary T. Hawn ${ }^{3,8}$, Yingjie Weng ${ }^{8}$, Amber W. Trickey ${ }^{8}$, Edward R. Mariano ${ }^{1,2}$ and Alex H. S. Harris 3,8

\begin{abstract}
Background: The American Society of Anesthesiologists Physical Status (ASA-PS) classification system was developed to categorize the fitness of patients before surgery. Increasingly, the ASA-PS has been applied to other uses including justification of inpatient admission. Our objectives were to develop and cross-validate a statistical model for predicting ASA-PS; and 2) assess the concurrent and predictive validity of the model by assessing associations between model-derived ASA-PS, observed ASA-PS, and a diverse set of 30-day outcomes.

Methods: Using the 2014 American College of Surgeons National Surgical Quality Improvement Program (ACS NSQIP) Participant Use Data File, we developed and internally cross-validated multinomial regression models to predict ASA-PS using preoperative NSQIP data. Accuracy was assessed with C-Statistics and calibration plots. We assessed both concurrent and predictive validity of model-derived ASA-PS relative to observed ASA-PS and 30-day outcomes. To aid further research and use of the ASA-PS model, we implemented it into an online calculator.

Results: Of the 566,797 elective procedures in the final analytic dataset, 8.9\% were ASA-PS 1, 48.9\% were ASA-PS 2, $39.1 \%$ were ASA-PS 3, and 3.2\% were ASA-PS 4. The accuracy of the 21 -variable model to predict ASA-PS was $C=$ $0.77+/-0.0025$. The model-derived ASA-PS had stronger association with key indicators of preoperative status including comorbidities and higher BMI (concurrent validity) compared to observed ASA-PS, but less strong associations with postoperative complications (predictive validity). The online ASA-PS calculator may be accessed at https://s-spire-clintools.shinyapps.io/ASA_PS_Estimator/

Conclusions: Model-derived ASA-PS better tracked key indicators of preoperative status compared to observed ASA-PS. The ability to have an electronically derived measure of ASA-PS can potentially be useful in research, quality measurement, and clinical applications.
\end{abstract}

Keywords: Anesthesiology/methods, Health status, Clinical prediction rule, Outcome assessment (health care), Reliability and validity

\section{Background}

The American Society of Anesthesiologists Physical Status Classification system (ASA-PS) is a commonly used, subjective method to categorize patients' fitness for surgery $[1,2]$. Originally developed by Saklad et al., the sixpoint classification system ranges from healthy patients

\footnotetext{
* Correspondence: mudumbai@stanford.edu

${ }^{1}$ Anesthesiology and Perioperative Care Service, Veterans Affairs Palo Alto Health Care System, 3801 Miranda Avenue, Palo Alto, CA 94402, USA

${ }^{2}$ Department of Anesthesiology, Perioperative and Pain Medicine, Stanford University School of Medicine, 291 Campus Drive, Stanford, CA 94305, USA Full list of author information is available at the end of the article
}

with no comorbidities (ASA-PS 1) to brain-dead patients whose organs are being removed for donor purposes (ASA-PS 6) [3]. Though the system was initiated more than 5 decades ago, the scoring system continues to perform fairly well in assessing patients for both inpatient and outpatient surgery [4].

While the original intent of the ASA-PS was to stratify severity of illness prior to surgery, more recently the ASA-PS has been used as a simple means to predict outcomes [5-7]. While other and potentially better surgical outcome prediction methods are available, most have

(c) The Author(s). 2019 Open Access This article is distributed under the terms of the Creative Commons Attribution 4.0 International License (http://creativecommons.org/licenses/by/4.0/), which permits unrestricted use, distribution, and reproduction in any medium, provided you give appropriate credit to the original author(s) and the source, provide a link to the Creative Commons license, and indicate if changes were made. The Creative Commons Public Domain Dedication waiver (http://creativecommons.org/publicdomain/zero/1.0/) applies to the data made available in this article, unless otherwise stated. 
been developed for specific surgical conditions rather than for 'surgery' in general [8]. The ASA-PS has face validity as an assessment of functional capacity, which is increasingly thought to be a significant predictor of patient outcome [9]. ASA-PS is now included within riskadjustment algorithms comparing hospital performance in surgical care i.e., the American College of Surgeons National Surgical Quality Improvement Program (ACS NSQIP) [10]. Models drawn entirely from preoperative NSQIP data may also be particularly helpful in risk stratification during the preoperative evaluation process $[7,11,12]$.

Though the ASA-PS has validity as a marker of patients' preoperative health status, multiple studies nevertheless have found that inter-rater reliability is moderate, meaning different anesthesiologists often give the same patient different classification levels $[2,13,14]$. Studies also indicate that ASA-PS may be missing or misclassified in data registries, which can lead to miscalculations of outcomes benchmarking for facilities $[15,16]$. Most concerning are ASA-PS scores that are far from their expected value given observed patient characteristics - for example a patient with a ASAPS IV but no recorded comorbidities. While ASA-PS as recorded in clinical databases may have measurement errors, an automated, risk model-derived calculation of ASA-PS that takes into account multiple aspects of the patients condition can serve as an initial proxy for improving and evaluating quality of care. The automated, risk model-derived ASA-PS may suggest more accurate initial values or corrections to these errors. Accordingly, the objectives of this study were to 1) develop and internally cross-validate a predictive model for ASA-PS using a wide range of preoperative predictors; and 2) assess the concurrent and predictive validity of the model by assessing associations between predicted ASA-PS, observed ASA-PS, and a diverse set of 30day outcomes.

\section{Methods}

\section{Data sources}

We used de-identified registry data; therefore, the study was exempt from IRB review. Our data source was the 2014 Participant Use File (PUF) of the American College of Surgeons National Surgical Quality Improvement Program (ACS-NSQIP), which is available to member institutions [17]. The ACS-NSQIP collects data on over 150 variables, including preoperative risk factors, ASA-PS, intraoperative variables, and 30-day postoperative mortality and morbidity outcomes for patients undergoing major surgical procedures in both the inpatient and outpatient surgical setting. Definitions for the variables are found within the PUF file.

\section{Development of analytic sample}

From the 750,937 surgeries represented in the 2014 PUF(Fig. 1), we excluded procedures that met any of the following criteria: emergent or non-elective surgeries; surgeries for patients transferred to the hospital (not admitted from home); patients $<18$ years old or $\geq 90$ years old at the time of surgery; procedures for patients who were inpatients immediately prior to their index surgery; surgeries for patients who had missing ASA assignment or had a ASA-PS class of '5-Moribund'; and procedures of patients who had incomplete data for any of the predictors identified for model development (see below). We selected these criteria given evidence of lower inter-rater agreement for ASA-PS for emergent cases or at extremes of age [14]. In addition, in trauma surgery, interrater reliability for assigning ASA-PS is fair at best and complete information on preoperative variables may be missing [18].

\section{Development and internal validation of a model to predict ASA-PS \\ Outcome}

Clinical assignment of ASA-PS status as represented in the 2014 NSQIP PUF on a scale from 1 to 4 (Table 1).

\section{Predictors}

We identified predictors that would be commonly available during preoperative evaluation such as demographics, codes indicative of common diagnoses and treatments, and functional status before surgery. We also evaluated preoperative laboratory data (i.e., pre-operative serum sodium) as potential predictors and noted that they were missing for a large proportion of cases, most likely not at random; and so did not include them in our final model.

- Sex: Defined as either male or female

- Race/Ethnicity: Defined with 6 categories: nonHispanic White; non-Hispanic Black; Hispanic; nonHispanic Asian; non-Hispanic Other; Unknown

- Age: Age of patient (18-89 yrs)

- Body mass index (BMI): Defined by height and weight by the following formula; (703 x wt (lbs) / (ht (in)) ${ }^{2}$

- Diagnoses and Treatments: - diabetes (None, insulin dependent; non-insulin dependent);

- hypertension required medication (yes, no; The patient must have been receiving or required longterm treatment of their chronic hypertension for $>2$ weeks.);

- chronic obstructive pulmonary disease (yes, no);

- congestive heart failure (yes, no);

- renal failure (yes, no);

- disseminated cancer (yes, no);

- smoker (yes, no);

- sepsis (none vs. any sepsis; septic shock; or

systemic inflammatory response syndrome (SIRS);

- ascites (yes, no);

- preoperative wound infection (yes, no); 


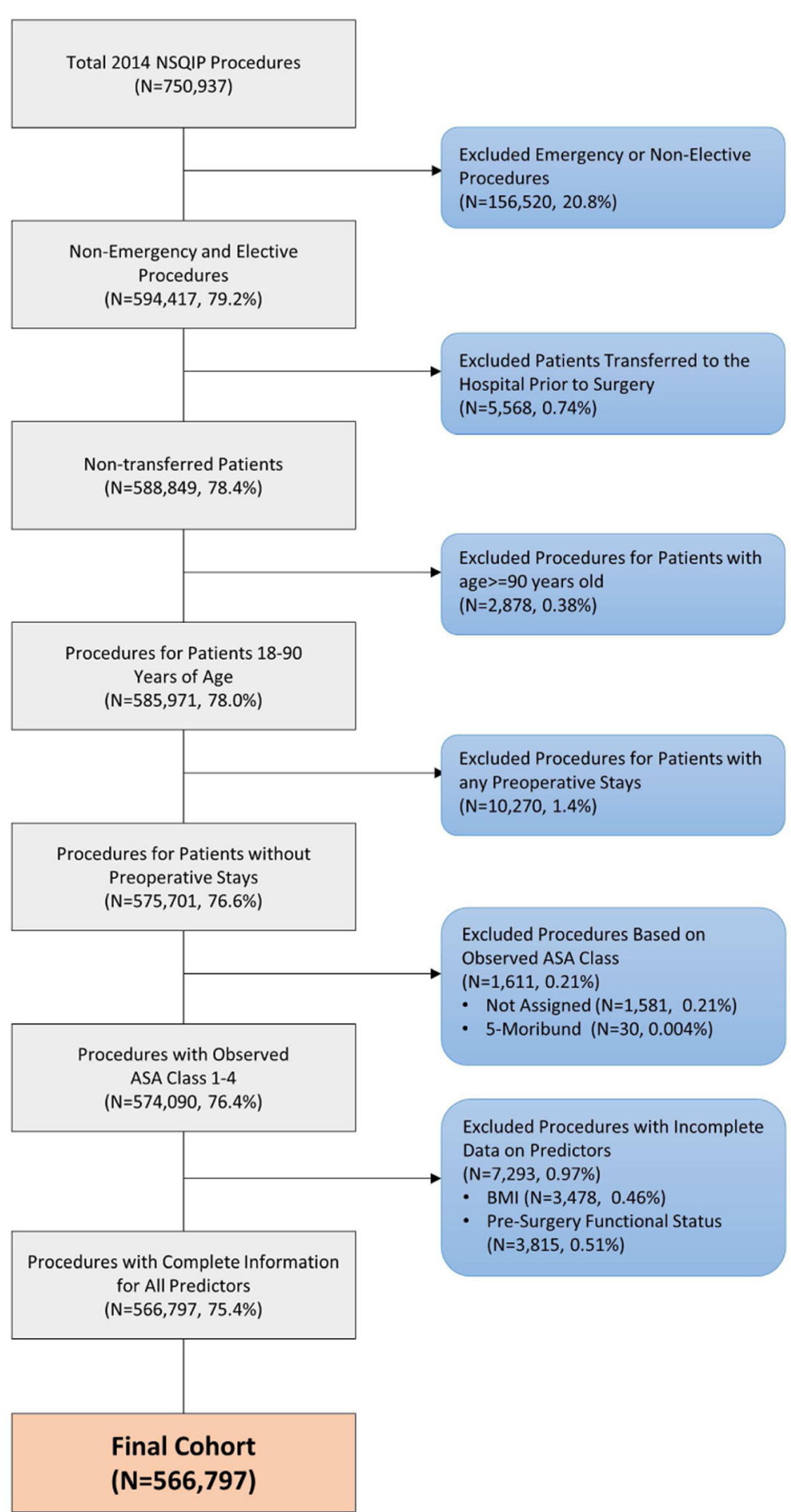

Fig. 1 Flowchart of Cohort Inclusion and Exclusion Criteria

- weight loss (yes, no);

- bleeding disorders (yes, no);

- dyspnea (none; at rest; or with moderate exertion);

- the presence of mechanical ventilation greater

than $48 \mathrm{~h}$ prior to surgery (yes, no);

- smoking status prior to surgery (yes, no);
- bleeding prior to surgery (yes, no),

- dialysis (yes, no);

- steroid use (yes, no);

- transfusion (yes, no);

- functional status before surgery (independent; partially dependent; totally dependent to perform 
Table 1 Current Definitions and Examples of American Society of Anesthesiologists Physical Status (ASA-PS) Class

\begin{tabular}{|c|c|c|}
\hline ASA Physical Status Classification & Definition & Examples, including, but not limited to: \\
\hline ASA-PS I & A normal healthy patient & Healthy, non-smoking, no or minimal alcohol use \\
\hline ASA-PS ॥ & $\begin{array}{l}\text { A patient with mild systemic disease } \\
\text { Mild diseases only without substantive } \\
\text { functional limitations. }\end{array}$ & $\begin{array}{l}\text { Mild diseases only without substantive functional } \\
\text { limitations. Examples include (but not limited to): } \\
\text { current smoker, social alcohol drinker, pregnancy, } \\
\text { obesity ( } 30<\text { BMl }<40 \text { ), well-controlled DM/HTN, } \\
\text { mild lung disease }\end{array}$ \\
\hline ASA-PS III & A patient with severe systemic disease & $\begin{array}{l}\text { Substantive functional limitations; One or more } \\
\text { moderate to severe diseases. Examples include } \\
\text { (but not limited to): poorly controlled DM or HTN, } \\
\text { COPD, morbid obesity (BMI } \geq 40 \text { ), active hepatitis, } \\
\text { Alcohol dependence or abuse, implanted } \\
\text { pacemaker, moderate reduction in ejection } \\
\text { fraction, ESRD undergoing regularly scheduled } \\
\text { dialysis, premature infant PCA }<60 \text { weeks, history } \\
\text { (>3 months) of MI, CVA, TIA, or CAD/stents. }\end{array}$ \\
\hline ASA-PS IV & $\begin{array}{l}\text { A patient with severe systemic disease } \\
\text { that is a constant threat to life }\end{array}$ & $\begin{array}{l}\text { Recent ( }<3 \text { months) history of MI, CVA, TIA, or } \\
\text { CAD/stents. Ongoing cardiac ischemia or severe } \\
\text { valve dysfunction, severe reduction of ejection } \\
\text { fraction, sepsis, DIC, ARD or ESRD not undergoing } \\
\text { regularly scheduled dialysis }\end{array}$ \\
\hline
\end{tabular}

$\begin{array}{ll}\text { ASA-PS V } & \text { A moribund patient who is not expected } \\ \text { to survive without the operation }\end{array}$

ASA-PS VI

A declared brain-dead patient whose organs

are being removed for donor purposes

Available at www.asahq.org/resources/clinical-information/asa-physical-status-classification-system; The addition of "E" denotes emergency surgery: (An emergency is defined as existing when delay in treatment of the patient would lead to a significant increase in the threat to life or body part)

activities of daily living [ADLs] in the 30 days prior to surgery).

\section{Statistical analysis}

Multinomial logistic regression was fitted on our final sample using ASA-PS as the outcome and variables listed above as predictors. The model was fitted using 'nnet' package built in R 3.3.0 [19]. We internally validated the model performance using 10-fold cross-validation. The C-statistic, an index of model accuracy, was calculated as the mean across the 10 repetitions. The $\mathrm{C}$-Statistic can be defined as the probability that a person who has been clinically assigned to a specific ASA classification has a higher predicted probability of being in that class than someone with a different classification [20, 21]. The C-statistic and its corresponding 95\% CI was calculated using the 'pROC' package in R. We compared the concurrent validity of predicted vs. observed ASA-PS (i.e., patients with predicted ASA-PS $>$ observed ASA-PS vs. those with observed ASA-PS > predicted ASAPS) by examining group associations with BMI, comorbidities, and functional status using two-sample Wilcoxon rank-sum (Mann-Whitney) tests.

\section{Assessing the predictive validity of the ASA-PS model Outcomes}

As defined in the ACS-NSQIP PUF, 30-day postoperative complications included the following binary (yes/no) outcome variables: mortality; and morbidity: venous thromboembolism; deep incisional surgical site infection; cardiac complications [myocardial infarction], cerebrovascular accident; respiratory complications [respiratory failure]; wound infections; sepsis; returned to the OR; renal insufficiency; and any complication.

\section{Predictors}

Coefficients from the multinomial logistical regression were used to predict the probabilities of each ASA-PS class (1-no disturbance, 2-mild, 3-severe, 4-life threat) for each person in the sample. We then assigned an ASA-PS class to each person based on their highest predicted probability (the class most likely) outputted from the model. In addition to the predicted ASA score, the following potential intraoperative confounders were included: Current Procedural Terminology (CPT) body system group (primary procedure codes were classified by major organ system type i.e., gastrointestinal, musculoskeletal etc., using Clinical Classification Software [CCS] systems); wound classification (clean/contaminated; contaminated; dirty/infected); anesthesia type (general anesthesia; spinal; epidural; monitored anesthesia care (MAC); or unknown); hospital length of stay in days; and operative time (total operative duration in hours) [22]. 


\section{Statistical analysis}

To compare the predictive power of observed vs. predicted ASA class, we first conducted separate logistic regressions using either as the independent variable and each 30-day postoperative complication as the outcome. C-statistics and the corresponding bootstrapped 95\% CI in each model were calculated and compared by the two ASA class types in parallel. In addition, we compared the C-statistics for each outcome adjusting for important intraoperative variables such as CPT body system group, wound classification, anesthesia type, hospital length of stay and operation type. We then further evaluated the predictive validity of our model by conducting a patient-level pairwise analysis i.e., a contingency table of predicted ASA-PS vs. observed ASAPS for each outcome (presence vs. absence).

We used SAS software, version 9.24 (SAS Institute Inc., Cary, NC, USA) and $\mathrm{R}$ software, version 3.0.2 (https://www.r-project.org/) for the statistical analyses, online tool development and graphics. To aid further research and use of the ASA-PS model, we implemented an online calculator using Shiny [23].

\section{Results}

\section{Sample characteristics}

Our final analytical dataset included a total of 566,797 elective procedures (Fig. 1 and Table 2). Overall, most of the patients (88\%) were ASA-PS $2(48.9 \%)$ or $3(39.1 \%)$, followed by ASA 1 (8.9\%) and ASA 4 (3.2\%). On average, the patients were in their mid-50s. The majority were female, obese (class I), predominantly white, mostly nondiabetic, hypertension requiring medications, with about one-fifth being smokers. Most of the patients did not have a diagnosis of COPD, CHF, renal failure, disseminated cancer, ascites, wound infections, weight loss, require transfusions, or having been on a ventilator.

Table 3 provides the most frequent procedures by CPT-body system classification and Top 20 level-one CCS (mapped by CPT) codes. The majority of patients had a digestive or musculoskeletal system procedure, though there were representative procedures across all other organ systems. The most frequent specific surgeries included those classified as other, hernia repairs, hysterectomy, and colorectal resections.

\section{Development and concurrent validation of a model to predict ASA-PS}

Additional file 1: Table S1 describes the details of our multinomial model with ASA-PS as an outcome. Across all ASA categories, conditions such as age, gender, race, or BMI were weakly predictive. However, conditions strongly predictive of ASA-PS status included total functional dependence, use of dialysis, insulin-dependent diabetes, disseminated cancer, COPD, hypertension treated with medications, and use of steroids.
Upon internal cross-validation, the overall C-statistic for the multinomial model (preoperative variables predicting observed ASA status) was C $=0.77$ (95\% CI: 0.766-0.773), signifying very good congruence between predicted and observed ASA status. The predicted ASA status agreed with the observed ASA-PS status for $99 \%$ of cases by one level (higher or lower) for ASA-PS $1-3$ and for $85 \%$ for ASA-PS 4. In general, our model tended to upgrade observed ASA I's to predicted ASA-PS II's. At the same time the model tended to downgrade observed ASA-PS IV's to predicted ASA-PS III's and V's to IV's and III's. This compression was most marked for observed ASA-PS IV. Preoperative factors associated with outliers for predicted ASA status revealed that in general discordances were found with extremes of age and BMI; procedures for musculoskeletal, nervous, and cardiovascular system CPT classes; and with diagnoses such as diabetes. In terms of concurrent validity, the group with predicted ASA-PS > observed ASA-PS had more comorbidities than patients with predicted ASA-PS < observed ASA-PS (mean 1.0 vs. $0.84, p<0.001$ ), higher BMI (30.7 vs. 29.6, $p<0.001$ ), and a trend toward more functional limitations (98.95\% independent vs. 99.03\% independent, $p=0.067$ ).

\section{Assessing the predictive validity of the ASA-PS model}

Using unadjusted, predicted ASA status to predict 30-day postoperative outcomes (predictive validity), the C-statistics ranged from $0.57-0.73$ with mortality at 0.73 (95\% CI: 0.719-0.740, Fig. 2). In comparison, using observed ASA status alone the C-statistics ranged from 0.58-0.76 with mortality at 0.73 (95\% CI: $0.719-0.740)$. The multivariable models (that included adjustment factors such as anesthesia type and operation time) increased the ability of the models to discriminate for the various outcomes with covariates explaining some of the variance in outcomes (Fig. 3). Higher C-statistics were noted for individual complications (i.e.,sepsis,renal insufficiency) vs. the category of any complication. Patients with predicted ASA-PS > observed ASAPS had a lower proportion of complications (3.4\% vs $6.7 \%$, $p<0.001)$ and lower mortality $(0.13 \%$ vs $0.41 \%, p<0.001)$.

Online risk calculator: The online calculator may be accessed at https://s-spire-clintools.shinyapps.io/ASA_PS_ Estimator/

\section{Discussion}

Our objective was to develop a model for predicting ASA-PS status using a wide range of preoperative predictors. We assessed both the concurrent and the predictive validity of predicted ASA-PS relative to observed ASA-PS and a variety of outcomes including 30-day morbidity and mortality. We noted that predicted ASAPS was more closely associated with key indicators of preoperative status including comorbidities and higher BMI with a trend towards functional status compared to 
Table 2 Characteristics of Study Sample

\begin{tabular}{|c|c|c|c|c|c|c|c|c|c|c|}
\hline & \multirow{2}{*}{$\begin{array}{l}\text { All } \\
N=566,797\end{array}$} & & \multicolumn{2}{|c|}{ ASA-PS ${ }^{a} 1$} & \multicolumn{2}{|c|}{ ASA-PS 2} & \multicolumn{2}{|c|}{ ASA-PS 3} & \multicolumn{2}{|c|}{ ASA-PS 4} \\
\hline & & & 50,285 & $8.9 \%$ & 277,156 & $48.9 \%$ & 221,453 & $39.1 \%$ & 17,903 & $3.2 \%$ \\
\hline & Avg & $\mathrm{sd}$ & Avg & sd & Avg & $\mathrm{sd}$ & Avg & $\mathrm{sd}$ & Avg & sd \\
\hline Age & 55.9 & 15.6 & 40.5 & 14.1 & 53.5 & 14.8 & 61.7 & 13.8 & 66.5 & 12.6 \\
\hline \multirow[t]{2}{*}{ BMI } & 30.5 & 7.7 & 26.5 & 4.8 & 29.5 & 6.4 & 32.7 & 8.8 & 31.2 & 9.5 \\
\hline & no. & $\%$ & no. & $\%$ & no. & $\%$ & no. & $\%$ & no. & $\%$ \\
\hline$n=$ & 566,797 & & 50,285 & $8.9 \%$ & 277,156 & $48.9 \%$ & 221,453 & $39.1 \%$ & 17,903 & $3.2 \%$ \\
\hline \multicolumn{11}{|l|}{ Sex } \\
\hline Female & 329,669 & $58.2 \%$ & 28,288 & $56.3 \%$ & 171,512 & $61.9 \%$ & 122,624 & $55.4 \%$ & 7245 & $40.5 \%$ \\
\hline Male & 237,128 & $41.8 \%$ & 21,997 & $43.7 \%$ & 105,644 & $38.1 \%$ & 98,829 & $44.6 \%$ & 10,658 & $59.5 \%$ \\
\hline \multicolumn{11}{|l|}{ Race/Ethnicity } \\
\hline White & 376,372 & $66.4 \%$ & 26,273 & $52.2 \%$ & 181,892 & $65.6 \%$ & 155,687 & $70.3 \%$ & 12,520 & $69.9 \%$ \\
\hline Black & 51,757 & $9.1 \%$ & 3502 & $7.0 \%$ & 23,248 & $8.4 \%$ & 22,967 & $10.4 \%$ & 2040 & $11.4 \%$ \\
\hline Hispanic & 37,982 & $6.7 \%$ & 4499 & $8.9 \%$ & 19,779 & $7.1 \%$ & 12,749 & $5.8 \%$ & 955 & $5.3 \%$ \\
\hline Asian & 14,675 & $2.6 \%$ & 2127 & $4.2 \%$ & 8103 & $2.9 \%$ & 4228 & $1.9 \%$ & 217 & $1.2 \%$ \\
\hline Other & 5156 & $0.9 \%$ & 420 & $0.8 \%$ & 2487 & $0.9 \%$ & 2135 & $1.0 \%$ & 114 & $0.6 \%$ \\
\hline Unknown & 80,855 & $14.3 \%$ & 13,464 & $26.8 \%$ & 41,647 & $15.0 \%$ & 23,687 & $10.7 \%$ & 2057 & $11.5 \%$ \\
\hline \multicolumn{11}{|l|}{ Dyspnea } \\
\hline No & 535,798 & $94.5 \%$ & 50,049 & $99.5 \%$ & 270,904 & $97.7 \%$ & 200,913 & $90.7 \%$ & 13,932 & $77.8 \%$ \\
\hline At Rest & 1517 & $0.3 \%$ & 12 & $0.0 \%$ & 226 & $0.1 \%$ & 954 & $0.4 \%$ & 325 & $1.8 \%$ \\
\hline Moderate Exertion & 29,482 & $5.2 \%$ & 224 & $0.4 \%$ & 6026 & $2.2 \%$ & 19,586 & $8.8 \%$ & 3646 & $20.4 \%$ \\
\hline \multicolumn{11}{|l|}{ Diabetes } \\
\hline No & 484,620 & $85.5 \%$ & 50,085 & $99.6 \%$ & 257,655 & $93.0 \%$ & 165,458 & $74.7 \%$ & 11,422 & $63.8 \%$ \\
\hline Non-Insulin & 54,427 & $9.6 \%$ & 159 & $0.3 \%$ & 15,501 & $5.6 \%$ & 35,767 & $16.2 \%$ & 3000 & $16.8 \%$ \\
\hline Insulin & 27,750 & $4.9 \%$ & 41 & $0.1 \%$ & 4000 & $1.4 \%$ & 20,228 & $9.1 \%$ & 3481 & $19.4 \%$ \\
\hline \multicolumn{11}{|l|}{ Sepsis } \\
\hline None & 564,736 & $99.6 \%$ & 50,176 & $99.8 \%$ & 276,369 & $99.7 \%$ & 220,454 & $99.5 \%$ & 17,737 & $99.1 \%$ \\
\hline Any Sepsis & 338 & $0.1 \%$ & 14 & $0.0 \%$ & 126 & $0.0 \%$ & 167 & $0.1 \%$ & 31 & $0.2 \%$ \\
\hline Septic Shock & 10 & $0.0 \%$ & 1 & $0.0 \%$ & 0 & $0.0 \%$ & 6 & $0.0 \%$ & 3 & $0.0 \%$ \\
\hline $\mathrm{SIRS}^{\mathrm{b}}$ & 1713 & $0.3 \%$ & 94 & $0.2 \%$ & 661 & $0.2 \%$ & 826 & $0.4 \%$ & 132 & $0.7 \%$ \\
\hline \multicolumn{11}{|l|}{ Functional Status } \\
\hline Independent & 561,050 & $99.0 \%$ & 50,238 & $99.9 \%$ & 276,133 & $99.6 \%$ & 217,680 & $98.3 \%$ & 16,999 & $95.0 \%$ \\
\hline Partially Dependent & 5321 & $0.9 \%$ & 47 & $0.1 \%$ & 976 & $0.4 \%$ & 3484 & $1.6 \%$ & 814 & $4.5 \%$ \\
\hline Totally Dependent & 426 & $0.1 \%$ & 0 & $0.0 \%$ & 47 & $0.0 \%$ & 289 & $0.1 \%$ & 90 & $0.5 \%$ \\
\hline \multicolumn{11}{|l|}{ Steroid Use } \\
\hline No & 547,932 & $96.7 \%$ & 50,114 & $99.7 \%$ & 271,015 & $97.8 \%$ & 210,236 & $94.9 \%$ & 16,567 & $92.5 \%$ \\
\hline Yes & 18,865 & $3.3 \%$ & 171 & $0.3 \%$ & 6141 & $2.2 \%$ & 11,217 & $5.1 \%$ & 1336 & $7.5 \%$ \\
\hline \multicolumn{11}{|c|}{ Hypertension with Medications } \\
\hline No & 312,618 & $55.2 \%$ & 49,344 & $98.1 \%$ & 183,331 & $66.1 \%$ & 76,345 & $34.5 \%$ & 3598 & $20.1 \%$ \\
\hline Yes & 254,179 & $44.8 \%$ & 941 & $1.9 \%$ & 93,825 & $33.9 \%$ & 145,108 & $65.5 \%$ & 14,305 & $79.9 \%$ \\
\hline \multicolumn{11}{|l|}{ Dialysis } \\
\hline No & 562,089 & $99.2 \%$ & 50,284 & $100.0 \%$ & 277,011 & $99.9 \%$ & 218,794 & $98.8 \%$ & 16,000 & $89.4 \%$ \\
\hline Yes & 4708 & $0.8 \%$ & 1 & $0.0 \%$ & 145 & $0.1 \%$ & 2659 & $1.2 \%$ & 1903 & $10.6 \%$ \\
\hline \multicolumn{11}{|l|}{$\mathrm{COPD}^{c}$} \\
\hline No & 544,439 & $96.1 \%$ & 50,252 & $99.9 \%$ & 274,216 & $98.9 \%$ & 205,483 & $92.8 \%$ & 14,488 & $80.9 \%$ \\
\hline
\end{tabular}


Table 2 Characteristics of Study Sample (Continued)

\begin{tabular}{|c|c|c|c|c|c|c|c|c|c|c|}
\hline \multirow{3}{*}{ Yes } & \multirow{2}{*}{$\begin{array}{l}\text { All } \\
N=566,797\end{array}$} & & \multicolumn{2}{|c|}{ ASA-PS ${ }^{a} 1$} & \multicolumn{2}{|c|}{ ASA-PS 2} & \multicolumn{2}{|c|}{ ASA-PS 3} & \multicolumn{2}{|c|}{ ASA-PS 4} \\
\hline & & & \multirow{2}{*}{$\begin{array}{l}50,285 \\
33\end{array}$} & \multirow{2}{*}{$\begin{array}{l}8.9 \% \\
0.1 \%\end{array}$} & \multirow{2}{*}{$\begin{array}{l}277,156 \\
2940\end{array}$} & \multirow{2}{*}{$\begin{array}{l}48.9 \% \\
1.1 \%\end{array}$} & \multirow{2}{*}{$\begin{array}{l}221,453 \\
15,970\end{array}$} & \multirow{2}{*}{$\begin{array}{l}39.1 \% \\
7.2 \%\end{array}$} & \multirow{2}{*}{$\begin{array}{l}17,903 \\
3415\end{array}$} & \multirow{2}{*}{$\frac{3.2 \%}{19.1 \%}$} \\
\hline & 22,358 & $3.9 \%$ & & & & & & & & \\
\hline \multicolumn{11}{|l|}{ Ascites } \\
\hline No & 565,885 & $99.8 \%$ & 50,278 & $100.0 \%$ & 276,930 & $99.9 \%$ & 220,878 & $99.7 \%$ & 17,799 & $99.4 \%$ \\
\hline Yes & 912 & $0.2 \%$ & 7 & $0.0 \%$ & 226 & $0.1 \%$ & 575 & $0.3 \%$ & 104 & $0.6 \%$ \\
\hline \multicolumn{11}{|c|}{ Congestive Heart Failure (CHF) } \\
\hline No & 564,691 & $99.6 \%$ & 50,282 & $100.0 \%$ & 277,071 & $100.0 \%$ & 220,210 & $99.4 \%$ & 17,128 & $95.7 \%$ \\
\hline Yes & 2106 & $0.4 \%$ & 3 & $0.0 \%$ & 85 & $0.0 \%$ & 1243 & $0.6 \%$ & 775 & $4.3 \%$ \\
\hline \multicolumn{11}{|c|}{ Renal Failure } \\
\hline No & 566,311 & $99.9 \%$ & 50,283 & $100.0 \%$ & 277,127 & $100.0 \%$ & 221,185 & $99.9 \%$ & 17,716 & $99.0 \%$ \\
\hline Yes & 486 & $0.1 \%$ & 2 & $0.0 \%$ & 29 & $0.0 \%$ & 268 & $0.1 \%$ & 187 & $1.0 \%$ \\
\hline \multicolumn{11}{|c|}{ Disseminated Cancer } \\
\hline No & 555,769 & $98.1 \%$ & 50,211 & $99.9 \%$ & 274,284 & $99.0 \%$ & 214,086 & $96.7 \%$ & 17,188 & $96.0 \%$ \\
\hline Yes & 11,028 & $1.9 \%$ & 74 & $0.1 \%$ & 2872 & $1.0 \%$ & 7367 & $3.3 \%$ & 715 & $4.0 \%$ \\
\hline \multicolumn{11}{|c|}{ Smoker } \\
\hline No & 469,380 & $82.8 \%$ & 46,060 & $91.6 \%$ & 230,441 & $83.1 \%$ & 179,155 & $80.9 \%$ & 13,724 & $76.7 \%$ \\
\hline Yes & 97,417 & $17.2 \%$ & 4225 & $8.4 \%$ & 46,715 & $16.9 \%$ & 42,298 & $19.1 \%$ & 4179 & $23.3 \%$ \\
\hline \multicolumn{11}{|c|}{ Wound Infection } \\
\hline No & 559,375 & $98.7 \%$ & 50,094 & $99.6 \%$ & 275,492 & $99.4 \%$ & 217,014 & $98.0 \%$ & 16,775 & $93.7 \%$ \\
\hline Yes & 7422 & $1.3 \%$ & 191 & $0.4 \%$ & 1664 & $0.6 \%$ & 4439 & $2.0 \%$ & 1128 & $6.3 \%$ \\
\hline \multicolumn{11}{|c|}{ Weight Loss } \\
\hline No & 561,766 & $99.1 \%$ & 50,214 & $99.9 \%$ & 275,688 & $99.5 \%$ & 218,311 & $98.6 \%$ & 17,553 & $98.0 \%$ \\
\hline Yes & 5031 & $0.9 \%$ & 71 & $0.1 \%$ & 1468 & $0.5 \%$ & 3142 & $1.4 \%$ & 350 & $2.0 \%$ \\
\hline \multicolumn{11}{|c|}{ Bleeding Disorder } \\
\hline No & 551,784 & $97.4 \%$ & 50,199 & $99.8 \%$ & 274,970 & $99.2 \%$ & 210,950 & $95.3 \%$ & 15,665 & $87.5 \%$ \\
\hline Yes & 15,013 & $2.6 \%$ & 86 & $0.2 \%$ & 2186 & $0.8 \%$ & 10,503 & $4.7 \%$ & 2238 & $12.5 \%$ \\
\hline \multicolumn{11}{|c|}{ Transfusion } \\
\hline No & 566,322 & $99.9 \%$ & 50,276 & $100.0 \%$ & 277,015 & $99.9 \%$ & 221,194 & $99.9 \%$ & 17,837 & $99.6 \%$ \\
\hline Yes & 475 & $0.1 \%$ & 9 & $0.0 \%$ & 141 & $0.1 \%$ & 259 & $0.1 \%$ & 66 & $0.4 \%$ \\
\hline \multicolumn{11}{|c|}{ Ventilator } \\
\hline No & 566,764 & $100.0 \%$ & 50,284 & $100.0 \%$ & 277,151 & $100.0 \%$ & 221,433 & $100.0 \%$ & 17,896 & $100.0 \%$ \\
\hline Yes & 33 & $0.0 \%$ & 1 & $0.0 \%$ & 5 & $0.0 \%$ & 20 & $0.0 \%$ & 7 & $0.0 \%$ \\
\hline
\end{tabular}

${ }^{\mathrm{a} A S A-P S}$ American Society of Anesthesiologists Physical Status, ${ }^{\mathrm{b}}$ SIRS Systemic inflammatory response syndrome, ${ }^{\mathrm{C}} \mathrm{COPD}$ Chronic obstructive pulmonary disease

observed ASA-PS on. The overall accuracy $(C=0.77)$ of our model was comparable to measurements of inter-rater reliability found when anesthesiologists evaluated preoperative physical status [2, 24-27]. Our study further highlights the advantages and challenges of using entirely preoperative data for risk calculation, especially as surgical risk models continue to be built $[7,8]$.

The ability to have an electronically derived measure of ASA-PS can potentially assist with research, quality measurement, resource allocation, and clinical applications. Studies indicate that higher ASA-PS patients can experience increased morbidity and mortality, higher rates of hospital readmissions, and costs when undergoing ambulatory surgery procedures [28-30]. With the aim of lowering surgical risks and improving patient outcomes, ASA-PS is increasingly being used by government bodies and insurance agencies to justify the need for hospital inpatient admission either for pre- or postoperative management [31, 32]. For example, preoperative hospital admission may be necessary for optimization of comorbidities like congestive heart failure; a postoperative admission instead may be indicated to avoid physiologic deterioration or to maintain functional status in the setting of surgical trauma. While 
Table 3 Surgery Characteristics of Study Sample

\begin{tabular}{|c|c|c|c|c|c|c|c|c|c|c|}
\hline & \multicolumn{2}{|l|}{ All } & \multicolumn{2}{|c|}{ ASA-PS 1} & \multicolumn{2}{|l|}{ ASA-PS 2} & \multicolumn{2}{|l|}{ ASA-PS 3} & \multicolumn{2}{|c|}{ ASA-PS 4} \\
\hline & no. & $\%$ & no. & $\%$ & no. & $\%$ & no. & $\%$ & no. & $\%$ \\
\hline$n=$ & 566,797 & & 50,285 & $8.9 \%$ & 277,156 & $48.9 \%$ & 221,453 & $39.1 \%$ & 17,903 & $3.2 \%$ \\
\hline \multicolumn{11}{|l|}{$\mathrm{CPT}^{\mathrm{a}}$ by Body System } \\
\hline Digestive System & 202,268 & $35.7 \%$ & 17,597 & $35.0 \%$ & 95,984 & $34.6 \%$ & 83,510 & $37.7 \%$ & 5177 & $28.9 \%$ \\
\hline Musculoskeletal System & 133,230 & $23.5 \%$ & 13,518 & $26.9 \%$ & 69,320 & $25.0 \%$ & 48,197 & $21.8 \%$ & 2195 & $12.3 \%$ \\
\hline Integumentary System & 55,656 & $9.8 \%$ & 6837 & $13.6 \%$ & 31,582 & $11.4 \%$ & 16,422 & $7.4 \%$ & 815 & $4.6 \%$ \\
\hline Female Genital System & 51,067 & $9.0 \%$ & 6211 & $12.4 \%$ & 32,055 & $11.6 \%$ & 12,433 & $5.6 \%$ & 368 & $2.1 \%$ \\
\hline Cardiovascular System & 33,281 & $5.9 \%$ & 982 & $2.0 \%$ & 4286 & $1.5 \%$ & 21,381 & $9.7 \%$ & 6632 & $37.0 \%$ \\
\hline Nervous System & 24,440 & $4.3 \%$ & 1399 & $2.8 \%$ & 12,114 & $4.4 \%$ & 10,322 & $4.7 \%$ & 605 & $3.4 \%$ \\
\hline Urinary System & 23,220 & $4.1 \%$ & 824 & $1.6 \%$ & 9606 & $3.5 \%$ & 11,847 & $5.3 \%$ & 943 & $5.3 \%$ \\
\hline Endocrine System & 20,993 & $3.7 \%$ & 1294 & $2.6 \%$ & 12,264 & $4.4 \%$ & 7009 & $3.2 \%$ & 426 & $2.4 \%$ \\
\hline Male Genital System & 10,173 & $1.8 \%$ & 954 & $1.9 \%$ & 5809 & $2.1 \%$ & 3303 & $1.5 \%$ & 107 & $0.6 \%$ \\
\hline Respiratory System & 6705 & $1.2 \%$ & 65 & $0.1 \%$ & 1460 & $0.5 \%$ & 4684 & $2.1 \%$ & 496 & $2.8 \%$ \\
\hline Hemic/lymphatic System & 3130 & $0.6 \%$ & 118 & $0.2 \%$ & 1392 & $0.5 \%$ & 1549 & $0.7 \%$ & 71 & $0.4 \%$ \\
\hline Auditory System & 1699 & $0.3 \%$ & 442 & $0.9 \%$ & 956 & $0.3 \%$ & 291 & $0.1 \%$ & 10 & $0.1 \%$ \\
\hline Mediastinum/Diaphragm & 821 & $0.1 \%$ & 12 & $0.0 \%$ & 250 & $0.1 \%$ & 501 & $0.2 \%$ & 58 & $0.3 \%$ \\
\hline Maternity Care/Delivery & 102 & $0.0 \%$ & 24 & $0.0 \%$ & 74 & $0.0 \%$ & 4 & $0.0 \%$ & 0 & $0.0 \%$ \\
\hline Reproductive System/Intersex & 12 & $0.0 \%$ & 8 & $0.0 \%$ & 4 & $0.0 \%$ & 0 & $0.0 \%$ & 0 & $0.0 \%$ \\
\hline General & 0 & $0.0 \%$ & 0 & $0.0 \%$ & 0 & $0.0 \%$ & 0 & $0.0 \%$ & 0 & $0.0 \%$ \\
\hline Eye/Ocular Adnexa & 0 & $0.0 \%$ & 0 & $0.0 \%$ & 0 & $0.0 \%$ & 0 & $0.0 \%$ & 0 & $0.0 \%$ \\
\hline \multicolumn{11}{|l|}{ Top 10 CPT CCS } \\
\hline Other & 165,715 & $29.2 \%$ & 17,769 & $35.3 \%$ & 69,833 & $25.2 \%$ & 69,032 & $31.2 \%$ & 9081 & $50.7 \%$ \\
\hline Other hernia repair & 45,057 & $7.9 \%$ & 4579 & $9.1 \%$ & 23,183 & $8.4 \%$ & 16,444 & $7.4 \%$ & 851 & $4.8 \%$ \\
\hline Arthroplasty knee & 39,155 & $6.9 \%$ & 941 & $1.9 \%$ & 19,391 & $7.0 \%$ & 18,192 & $8.2 \%$ & 631 & $3.5 \%$ \\
\hline Hysterectomy, abdominal and vaginal & 37,837 & $6.7 \%$ & 3989 & $7.9 \%$ & 24,025 & $8.7 \%$ & 9532 & $4.3 \%$ & 291 & $1.6 \%$ \\
\hline Colorectal resection & 32,851 & $5.8 \%$ & 891 & $1.8 \%$ & 15,974 & $5.8 \%$ & 15,097 & $6.8 \%$ & 889 & $5.0 \%$ \\
\hline Inguinal and femoral hernia repair & 26,881 & $4.7 \%$ & 4960 & $9.9 \%$ & 15,055 & $5.4 \%$ & 6401 & $2.9 \%$ & 465 & $2.6 \%$ \\
\hline Cholecystectomy & 26,553 & $4.7 \%$ & 3282 & $6.5 \%$ & 15,791 & $5.7 \%$ & 7111 & $3.2 \%$ & 369 & $2.1 \%$ \\
\hline Gastric bypass & 24,679 & $4.4 \%$ & 110 & $0.2 \%$ & 6890 & $2.5 \%$ & 17,091 & $7.7 \%$ & 588 & $3.3 \%$ \\
\hline Hip replacement & 24,094 & $4.3 \%$ & 964 & $1.9 \%$ & 13,123 & $4.7 \%$ & 9556 & $4.3 \%$ & 451 & $2.5 \%$ \\
\hline Lumpectomy & 22,100 & $3.9 \%$ & 2774 & $5.5 \%$ & 12,913 & $4.7 \%$ & 6157 & $2.8 \%$ & 256 & $1.4 \%$ \\
\hline
\end{tabular}

${ }^{\mathrm{a} C P T}$ Current Procedural Terminology, ${ }^{\mathrm{b}}$ Clinical Classification Software

observed ASA-PS correlated more strongly with 30-day outcomes than predicted ASA-PS in our study, predicted ASA-PS values could be useful in situations where a pre-populated estimate in an EHR is needed (see Shiny application) or in studies where some or all cases are missing ASA-PS values [33]. Alignment of provider or facility decisions with government or payor guidelines can then be evaluated post-hoc for sample stratification where ASA-PS is missing and for quality monitoring. Calculation of a predicted ASA-PS could also aid in care coordination at the time of initial preoperative evaluation $[5,7,14,34]$. Guidelines suggest that nonanesthesia clinicians when faced with the need to provide moderate or deep sedation for ASA-PS III or IV should have an anesthesia clinician present to avoid adverse outcomes such as respiratory arrest [35]. An automated process for calculating ASA-PS may therefore be helpful in generating electronic prompts and reminders.

Our study extends methods and results by Davenport et al. that evaluated the relationship between predicted ASA-PS, other preoperative risk factors, and 30- day morbidity and mortality outcomes [36]. While our study was a national level sample, Davenport's study used $\mathrm{Na}$ tional Surgical Quality Improvement Project data for 5878 surgical patients at a university medical center; they similarly noted that observed ASA-PS was a stronger predictor of 30-day outcomes than predicted ASAPS. A potential explanation for these types of 


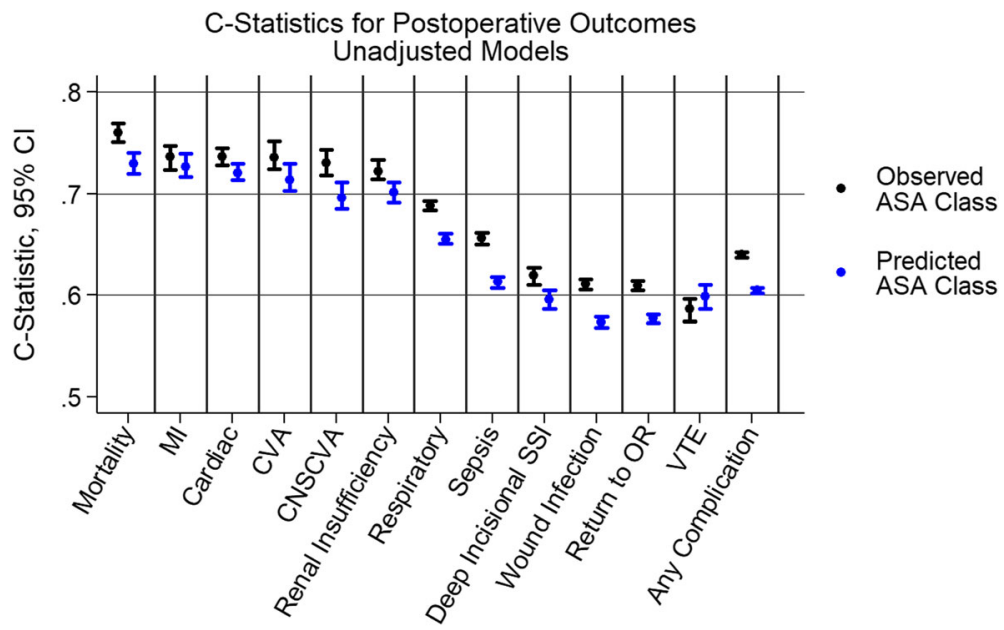

Fig. 2 C-Statistics for Post-Operative Outcomes (Unadjusted)

discordances is that ASA-PS remains a subjective measure of disease status and prone to differences in opinion when offered discrete categories (i.e.1,2). While the discrimination of our multivariable adjusted models in predicting 30-day outcomes was better than with univariate models, our results like Davenport's suggest the further need for intraoperative variables and facility level data for risk adjustment. The fact that observed ASA-PS correlated more strongly with 30-day outcomes than predicted ASA -PS may reflect the fact that there are other confounding variables that still need to be accounted for in our predictive model. A prominent category of variables that we were unable to include was patients' preoperative laboratory data; laboratory data was found to be not missing at random (NMAR) in our sample. In their predictive model for ASA-PS, Davenport et al. found that laboratory measures such as low serum albumin, high white blood cell count, and low hematocrit were strong predictors of 30-day outcomes [36]. The ability to include preoperative laboratory data could further strengthen the predictive validity of our model.

\section{Limitations and strengths}

Given that our study involved the use of preexisting data, there are certain limitations and strengths. The analysis of preexisting data is always limited by its extant quality, the type of elements that it collects and the number of facilities. However, our study sample was a national cohort drawn from a broad variety of facilities across a wide geographic distribution. We also evaluated multiple sociodemographic criteria along with detailed clinical data and administrative data to enhance external validity. Another potential limitation is that while the

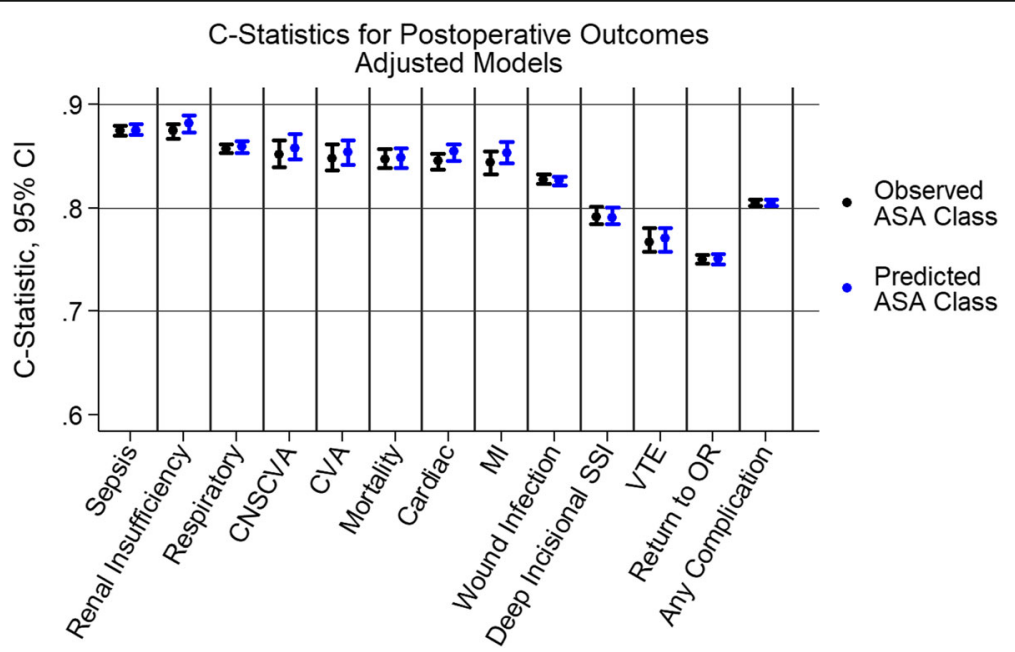

Fig. 3 C-Statistics for Post-Operative Outcomes (Adjusted) 
ACS-NSQIP data set captures a wide variety of cases, nevertheless, it is still not $100 \%$ case capture. Nevertheless, our sample included a diverse set of procedures with detailed clinical data and administrative data that enhanced the concurrent validity of our models. As a national, clinical registry, the ACS-NSQIP is bound to contain some level of error or inconsistency. We attempted to exclude patients with a preoperative stay due to concerns that a preoperative stay might indicate the presence of conditions (like myocardial infarction) or events (need for preoperative optimization) that might enter into determination of ASA-PS. In addition, these conditions may not necessarily be observable through the ACS-NSQIP database. However, even with this exclusion, a very small number of patient with other indicators suggesting a preoperative stay, such as the 33 patients (out of the total sample of 566,797) who were on a ventilator in the $48 \mathrm{~h}$ prior to surgery, were not excluded. Given the very rare nature of these apparent errors or inconsistencies, we maintain that any potential bias would be negligible.

\section{Conclusions}

The ability to have an electronically-derived measure of ASA-PS can assist with resource allocation and quality measurement as well as care coordination [33].. The model- based approach demonstrated here appears to be equal to or more valid than existing holistic judgments. An important key challenge is to demonstrate how these automatic approaches might be accepted by clinicians and actually add value. It has been widely published in the decision making literature that statistical models outperform crude judgments [37]. The original ASA-PS score, despite being a 'quick-and-dirty' tool, is easy to use and easy to apply. Approaches that combine subjective judgments and objective information may outperform both and should be evaluated as part of an implementation study. In addition, by enabling the prediction of ASA-PS when it is not available and potentially improving the measure's accuracy when ASA-PS is available, we also see our effort as an aid for other researchers and clinicians to build better risk prediction models. Further external validation of this model with non-ACS-NSQIP data will help define the minimal number of elements needed to predict ASA-PS and further refine predictive ability $[26,38,39]$.

\section{Supplementary information}

Supplementary information accompanies this paper at https://doi.org/10. 1186/s12913-019-4640-x.

Additional file 1: Table S1. Coefficients for the Multinomial Model to Predict ASA Class by Major Pre-operative Factors.

\section{Abbreviations}

ACS NSQIP: American College of Surgeons National Surgical Quality Improvement Program; ASA-PS: American Society of Anesthesiologists Physical Status Classification system; BMI: Body mass index; CCS: Clinical Classification Software; MAC: Monitored anesthesia care; PUF: Participant Use File; SIRS: Systemic inflammatory response syndrome

\section{Acknowledgements}

None.

\section{Authors' contributions}

Every author below is has made substantial contributions to the conception and design of the work; the analysis and interpretation of data; has drafted the work or substantively revised it; and has approved the submitted version; has agreed both to be personally accountable for the author's own contributions and to ensure that questions related to the accuracy or integrity of any part of the work, even ones in which the author was not personally involved, are appropriately investigated, resolved, and the resolution documented in the literature. All authors read and approved the final manuscript. Authors: SCM; SP; TB; RK; EDS; AKF; DE; MH; YW; AWT;ERM,AHS

\section{Funding}

This work was funded by grants from the VA HSR\&D Service (IIR 16-216; RCS14-232). The funding body had no role in the design of the study and data collection, analysis, and interpretation of data and in writing of the manuscript.

\section{Availability of data and materials}

Our data source was the 2014 Participant Use File (PUF) of the American College of Surgeons National Surgical Quality Improvement Program (ACSNSQIP), which is available to member institutions.

\section{Ethics approval and consent to participate}

We used publically available registry data from the American College of Surgeons National Surgical Quality Improvement Program (ACS-NSQIP) program. The de-identified dataset does not contain any data or code or link that could allow identification of the individual. As defined by federal regulations, 45 CFR 46.102, this is not deemed human subject research and exempt from IRB review and informed consent.

Consent for publication

Not applicable.

\section{Competing interests}

The authors declare that they have no competing interests.

\section{Author details}

${ }^{1}$ Anesthesiology and Perioperative Care Service, Veterans Affairs Palo Alto Health Care System, 3801 Miranda Avenue, Palo Alto, CA 94402, USA.

${ }^{2}$ Department of Anesthesiology, Perioperative and Pain Medicine, Stanford University School of Medicine, 291 Campus Drive, Stanford, CA 94305, USA. ${ }^{3}$ Center for Innovation to Implementation, Veterans Affairs Palo Alto Health Care System, 795 Willow Road (152-MPD), Menlo Park, California 94025, USA. ${ }^{4}$ Department of Ophthalmology, Stanford University School of Medicine, 291 Campus Drive, Stanford, CA 94305, USA. ${ }^{5}$ Department of Orthopaedic Surgery, Stanford University School of Medicine, 291 Campus Drive, Stanford, CA 94305, USA. ${ }^{6}$ Department of Surgery, Section of Plastic Surgery at the University of Michigan, 2101 Taubman Center 1500 E. Medical Center Drive, Ann Arbor, Ml 48109, USA. ${ }^{7}$ Center for Clinical Management Research, VA Ann Arbor Healthcare System, 2215 Fuller Rd, Ann Arbor, MI 48105, USA. ${ }^{8}$ Department of Surgery Surgery Policy Improvement Research and Education (S-SPIRE) Center, Stanford University School of Medicine, 291 Campus Drive, Stanford, CA 94305, USA. 
Received: 28 August 2019 Accepted: 15 October 2019

Published online: 21 November 2019

\section{References}

1. ASA Physical Status Classification System, October 2014. Available at: https:// www.asahq.org/resources/clinical-information/asa-physical-statusclassification-system. Accessed Jan 52018

2. Owens WD, Felts JA, Spitznagel EL Jr. ASA physical status classifications: a study of consistency of ratings. Anesthesiology. 1978;49(4):239-43.

3. Saklad M. Grading of patients for surgical procedures. Anesthesiology. 1941; 2(3):281-4.

4. Keats AS. The ASA classification of physical status--a recapitulation. Anesthesiology. 1978;49(4):233-6.

5. Wolters U, Wolf T, Stutzer H, Schroder T. ASA classification and perioperative variables as predictors of postoperative outcome. Br J Anaesth. 1996;77(2):217-22.

6. Reponen $\mathrm{E}$, Tuominen $\mathrm{H}$, Korja M. Evidence for the use of preoperative risk assessment scores in elective cranial neurosurgery: a systematic review of the literature. Anesth Analg. 2014;119(2):420-32.

7. Moonesinghe SR, Mythen MG, Das P, Rowan KM, Grocott MP. Risk stratification tools for predicting morbidity and mortality in adult patients undergoing major surgery: qualitative systematic review. Anesthesiology. 2013;119(4):959-81.

8. Peacock O, Bassett MG, Kuryba A, Walker K, Davies E, Anderson I, Vohra RS, National Emergency Laparotomy Audit Project T. Thirty-day mortality in patients undergoing laparotomy for small bowel obstruction. Br J Surg. 2018;105(8):1006-13.

9. Mayhew D, Mendonca V, Murthy BVS. A review of ASA physical status - historical perspectives and modern developments. Anaesthesia. 2019;74(3):373-9.

10. Cohen ME, Dimick JB, Bilimoria KY, Ko CY, Richards K, Hall BL. Risk adjustment in the American College of Surgeons National Surgical Quality Improvement Program: a comparison of logistic versus hierarchical modeling. J Am Coll Surg. 2009;209(6):687-93.

11. Bilimoria KY, Liu Y, Paruch JL, Zhou L, Kmiecik TE, Ko CY, Cohen ME. Development and evaluation of the universal ACS NSQIP surgical risk calculator: a decision aid and informed consent tool for patients and surgeons. J Am Coll Surg. 2013;217(5):833-842.e831-833.

12. Harris AH, Kuo AC, Bowe T, Gupta S, Nordin D, Giori NJ. Prediction models for 30-day mortality and complications after Total knee and hip Arthroplasties for veteran health administration patients with osteoarthritis. J Arthroplast. 2018;33(5):1539-45.

13. Curatolo C, Goldberg A, Maerz D, Lin HM, Shah H, Trinh M. ASA physical status assignment by non-anesthesia providers: do surgeons consistently downgrade the ASA score preoperatively? J Clin Anesth. 2017;38:123-8.

14. Sankar A, Johnson SR, Beattie WS, Tait G, Wijeysundera DN. Reliability of the American Society of Anesthesiologists physical status scale in clinical practice. Br J Anaesth. 2014;113(3):424-32.

15. Helkin A, Jain SV, Gruessner A, Fleming M, Kohman L, Costanza M, Cooney RN. Impact of ASA score misclassification on NSQIP predicted mortality: a retrospective analysis. Perioper Med (Lond). 2017;6:23.

16. Liau A, Havidich JE, Onega T, Dutton RP. The National Anesthesia Clinical Outcomes Registry. Anesth Analg. 2015;121(6):1604-10.

17. See 2014 ACS NSQIP DATA USER GUIDE . Accessed through https://www. facs.org/quality-programs/acs-nsqip/participant-use . 5 Jan 2017.

18. Kuza CM, Hatzakis G, Nahmias JT. The assignment of American Society of Anesthesiologists Physical Status Classification for adult Polytrauma patients: results from a survey and future considerations. Anesth Analg. 2017;125(6):1960-6.

19. Venables WN, Ripley BD. Modern applied statistics with S. 4th ed. New York: Springer; 2002. ISBN 0-387-95457-0

20. Robin X, Turck N, Hainard A, Tiberti N, Lisacek F, Sanchez JC, Muller M. PROC: an open-source package for $\mathrm{R}$ and $\mathrm{S}+$ to analyze and compare ROC curves. BMC Bioinformatics. 2011;12:77.

21. Hand DJ, Till RJ. A simple generalisation of the area under the ROC curve for multiple class classification problems. Mach Learn. 2001;45(2):171-86.

22. Elixhauser A, Steiner C, Palmer L. Clinical classifications software (CCS), 2014. US Agency for Healthcare Research and Quality Available at https://www. hcup-us.ahrq.gov/toolssoftware/ccs/ccs.jsp. Accessed 4 May 2018.

23. Chang W, Cheng J, Allaire J, Y. X, McPherson J: shiny: Web Application Framework for R. R package version 1.2.0 https://CRAN.R-project.org/package=shiny. In.; 2018. Accessed 12 Nov 2018.

24. Ihejirika RC, Thakore RV, Sathiyakumar V, Ehrenfeld JM, Obremskey WT, Sethi MK. An assessment of the inter-rater reliability of the ASA physical status score in the orthopaedic trauma population. Injury. 2015;46(4):542-6.
25. Hurwitz EE, Simon M, Vinta SR, Zehm CF, Shabot SM, Minhajuddin A, Abouleish AE. Adding examples to the ASA-physical status classification improves correct assignment to patients. Anesthesiology. 2017;126(4):614-22.

26. Haynes SR, Lawler PG. An assessment of the consistency of ASA physical status classification allocation. Anaesthesia. 1995;50(3):195-9.

27. Dexter F. Importance of relying on examples for both anesthesiologists and other physicians to assign unbiased American Society of Anesthesiologists Physical Status Classifications. J Clin Anesth. 2017;39:118-9.

28. Whippey A, Kostandoff G, Paul J, Ma J, Thabane L, Ma HK. Predictors of unanticipated admission following ambulatory surgery: a retrospective casecontrol study. Can J Anaesth. 2013;60(7):675-83.

29. Rao A, Polanco A, Qiu S, Kim J, Chin EH, Divino CM, Nguyen SQ. Safety of outpatient laparoscopic cholecystectomy in the elderly: analysis of 15,248 patients using the NSQIP database. J Am Coll Surg. 2013;217(6):1038-43.

30. Fleisher LA, Pasternak LR, Herbert R, Anderson GF. Inpatient hospital admission and death after outpatient surgery in elderly patients: importance of patient and system characteristics and location of care. Arch Surg. 2004; 139(1):67-72.

31. Massachusetts Medical Society. Chapter IV: patient admission and discharge. Office-Based Surgery Guidelines. Waltham, MA: Massachusetts Medical Society; 2011:11. www.massmed.org/officebasedsurgery/. Accessed 1 June 2018.

32. AlM Specialty Health. Preoperative Admission Guidelines for Musculoskeletal Surgery and Procedures http://aimspecialtyhealth.com/PDF/Guidelines/2018/ Mar01/AIM_Guidelines_MSK_PreoperativeAdmission.pdf Accessed 1 June 2018.

33. Eakin JL, Bader AM. ASA physical status classification system: is it consistent amongst providers and useful in determining need for pre-operative evaluation resources? J Clin Anesth. 2017;39:73-4.

34. Protopapa KL, Simpson JC, Smith NC, Moonesinghe SR. Development and validation of the surgical outcome risk tool (SORT). Br J Surg. 2014;101(13):1774-83.

35. American Society of Anesthesiologists Task Force on S, Analgesia by N-A. Practice guidelines for sedation and analgesia by non-anesthesiologists. Anesthesiology. 2002;96(4):1004-17.

36. Davenport DL, Bowe EA, Henderson WG, Khuri SF, Mentzer RM Jr. National Surgical Quality Improvement Program (NSQIP) risk factors can be used to validate American Society of Anesthesiologists Physical Status Classification (ASA PS) levels. Ann Surg. 2006;243(5):636-41 discussion 641-634.

37. Grove WM, Zald DH, Lebow BS, Snitz BE, Nelson C. Clinical versus mechanical prediction: a meta-analysis. Psychol Assess. 2000;12(1):19-30.

38. Avidan A, Weiniger CF. Keep American Society of Anesthesiologists Physical Status Classification System Simple, Stupid. Anesthesiology. 2018;128(1):225-6.

39. Harris AH. Path from predictive analytics to improved patient outcomes: a framework to Guide use, implementation, and evaluation of accurate surgical predictive models. Ann Surg. 2017;265(3):461-3.

\section{Publisher's Note}

Springer Nature remains neutral with regard to jurisdictional claims in published maps and institutional affiliations.

Ready to submit your research? Choose BMC and benefit from:

- fast, convenient online submission

- thorough peer review by experienced researchers in your field

- rapid publication on acceptance

- support for research data, including large and complex data types

- gold Open Access which fosters wider collaboration and increased citations

- maximum visibility for your research: over $100 \mathrm{M}$ website views per year

At BMC, research is always in progress.

Learn more biomedcentral.com/submissions 